\title{
Designing typefaces for maps. A protocol of tests.
}

\author{
Sébastien Biniek, ${ }^{a}$ Guillaume Touya, ${ }^{a}$ Gilles Rouffineau, ${ }^{b}$ and Thomas Huot-Marchand ${ }^{\mathrm{c}}$ \\ ${ }^{a}$ Univ. Paris-Est, LASTIG COGIT, IGN, ENSG, F-94160 Saint-Mande, France \\ ${ }^{b}$ ÉSAD Grenoble Valence, 38 000, Grenoble, France \\ ${ }^{c}$ ANRT, ENSAD, 54013, Nancy, France.
}

\begin{abstract}
The text management in map design is a topic generally linked to placement and composition issues. Whereas the type design issue is rarely addressed or at least only partially. Moreover the typefaces especially designed for maps are rare. This paper presents a protocol of tests to evaluate characters for digital topographic maps and fonts that were designed for the screen through the use of geographical information systems using this protocol. It was launched by the Atelier National de Recherche Typographique Research (ANRT, located in Nancy, France) and took place over his 'post-master' course in 2013. The purpose is to isolate different issues inherent to text in a topographic map: map background, nonlinear text placement and toponymic hierarchies. Further research is necessary to improve this kind of approach.
\end{abstract}

Keywords: Typography, Fonts, Topographic maps

\section{Introduction}

The graphic and textual components of a map form a single image that is both seen and read but unlike map objects, map texts doesn't have pre-defined positions. On the other hand, the text is always linked to a map object which he indicated. This implies taking into account cartographic signs, both to clearly understand which element is designated but also because it is necessary to avoid masking others. (Barrault 2001)

In other words, the text is a layer that overlays the background map while interacting with it. Creating this layer properly can be hard to manage. Indeed typesetting the text manually is a difficult and long task because it leave to the operator a relatively substantial leeway, in spite of the existence of cartographic rules. Placing labels inside a map is a task that requires to make some decisions regarding three key issues: text placement, text content and text forms. Text content refers to choosing which objects are to be displayed in text form. Then you have to define the actual string of characters to be used to typeset the target toponym. Text form involves making choices among such text variables as font face, size and color. In the past decades, the automation of text placement and typeset-ting has been one of the main goal in type matters and has still a forefront place in the debate.

It is mainly for these reasons that the text management in map design is a topic generally linked to placement and composition issues. Whereas the type design issue is rarely addressed or at least only partially. Moreover the typefaces especially designed for maps are rare. One might mention at this point as an exception: the Cisalpin font family designed by Felix Arnold in 2004. Furthermore, it is really difficult to find a paper devoted to the theme in the typographic literature (Bartz 1970). It cannot be said that cartography is used to exploit the latest innovations in type design. Even some typographic choices can be disappointing for type designers. For example, we would like to notice the recent change of typeface that has been operated at Swisstopo (the Swiss national mapping agency). It is the first time that a sansserif typeface appears in swiss maps. What is particularly striking in this example is that their choice does not reflect the strong typographic culture of Switzerland. In the years of economic expansion -past the aftermath of 1945 - Switzerland was one of the countries where the culture of sans-serif typography had the strongest impact. This country was the leading model of modern typography. It has taken more than 50 years for Swiss to use modern letterforms in their maps. It shows how this disciplines can be disconnected.

This paper presents a protocol of tests to evaluate characters for digital topographic maps and a typeface designed in parallel. This project was launched by the Atelier National de Recherche Typographique Research (ANRT, located in Nancy, France). It has been originally named : 'Map making, new topology' just intended to design a typeface for digital mapping. One of the first things to do was to define a more specific purpose. Topographic maps are an obvious choice as a use case because they are intended to providing information on the diversity of the territory. By definition, topography tries to give an objective picture of the relationships between man and his environment at a given scale ; endeavoring to provide a readable description of natural forms (vegetation, topography, hydrography, etc..) and artificial forms (buildings, roads and other communications) so that everyone can imagine the landscape according to their requirements.

By showing geographical objects and closely matching/fitting his typesetting to the shapes of the 
landscape, map text founds his uniqueness. It is basically an action of landscape forces on formal abstractions of language. It can indicate a position, an extended geographical area or a path. In many ways, it is a different paradigm or a way of understanding text, which is traditionally typeset in linear text bloc. Beyond its purely poetic aspect, this particular relationship between the text and the territory can provide a fertile and vital field of study for a type design project. Moreover this thematic is rarely addressed.

\section{Project description}

\subsection{State of the art and documented corpus}

It was not clear from the outset that a protocol would have to be built. First, the process consisted in studying the typographic practices used in different maps. Two main scales were studied: $1: 25 \mathrm{k}$ and $1: 50 \mathrm{k}$. We built a corpus of maps comprising mostly IGN (the French national mapping agency) printed maps, from the $20 \mathrm{~s}$ to today. We were interested in what kind of typographic choices were made in the successive generations of the IGN's standards and how these choices have been defined? It helped us to understand how typographic hierarchies in maps are constructed in terms of composition, spatialization and systematization from an historical perspective. We tried to understand how different levels of information coexist to allow the building of a coherent cartographic message.

\subsection{Definition issue}

The choice of such period was also made in view of analyzing the overall evolution of typesetting in French cartography. We were interested in what happens when cartographers have stopped using engraved or drawn letter-forms to typeset the toponyms. As type designers we believed that distinction makes sense. According to Gerrit Noordzij (Noordzij 2000) 'typography is writing with prefabricated letters'. His definition and his theory that give rise to it, suggest a smart and reflective articulation between typography and writing. He found typography in the practice of writing, while keeping it seperate. According to Noordzij $(2005,9)$ :

From a typographic point of view, type is a special branch of writing that differs essentially from lettering. The typographer can only work with writing that is arranged in a font. Since we learned to store typefaces in computers we can imagine type as lettering reproduced in a database (the typographic 'font') that makes the shapes of the original drawings available for composition. Lettering does not meet this typesetter's condition by itself.

This clarification allows to distinguish between these two notions, which can sometimes be expressed confusedly in the typographic culture. In France, for example, the term "écriture" - which can imply a scriptural dimension - is often used to express typographical notions.

Beyond confusion and inaccuracy, perhaps this indicates, in cartography, a more flexible or empirical approach of letters, because this discipline require them to fit in particular setting contexts.

\subsection{The method of analysis}

The methods were largely descriptive, trying to focus on critical phenomena and analysis the structure of letter shapes. You can construe letters and words from a structural point of view, either by describing the contrast and the kind of construction as Noordzij's precepts or using a settings method as Typecooker (a tool for typedrawing), but it is always helpful to be able to identify properly the typefaces. That why we completed this corpus with some documents as specifications (Figure 1), former cartographic reports and type specimens. Moreover this information is rarely collected. If this work isn't usually undertaken and researchers in cartography are few to tackle this issue when it comes to defining cartographic styles, it is because they do not have access to the information or because they do not have the capacity to analyze and use it. It is also possible that they are not even aware of their utility.

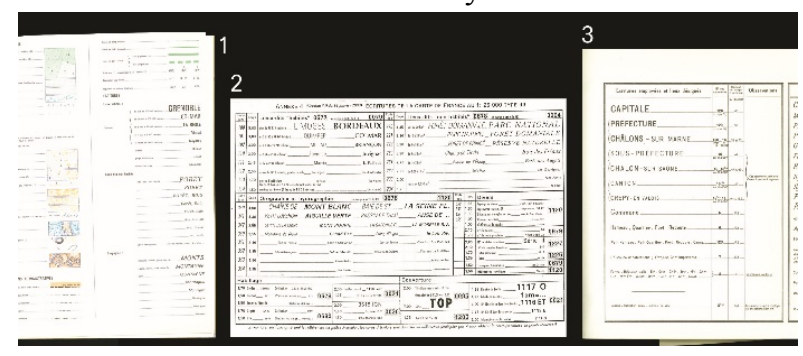

Fig. 1. Scans of typographic standards/specifications of IGN. 1: Type 1972, 2: Type 1993, 3: Type 1922.

By using this approach and correlating maps and documents we made the following observations:

- The hydronyms are very massively typeset in italic. This differentiation does not raise any major problem of legibility. In the long run, it has become a conventional process in map reading that cannot be overlooked. So it is important to make it apply and to ensure that it continues, insofar as it facilitates rapid identification of this category.

- The open shapes naturally leave more space to the map background and consequently prevent them from closing and clogging in places which would make impossible the recognition of letter shapes. Letterforms also must be distinguishable, to avoid any misunderstandings.

- Some toponyms, like small towns or street names are typeset in very small size, therefore it is important to increase the legibility of their letters.

- Some details in typefaces, like square dots on the "i", may cause some drawbacks. They can be confused with buildings blocks.

- In some printed maps there might be a competition between standards because of a simultaneous use, even though it is an increasingly rare phenomenon noticed. 
We think this kind of analysis is always useful to characterize some of cartographic practices. Actually, it is still a marginal approach.

This mission has not been completed to afford a more prominent place to the type design process and the development of the protocol. It requires a more comprehensive inquiry. We still have to identify some fonts that have been used and understand some processes linked to printed matters. We expect that approach will benefit both disciplines.

\subsection{The protocol}

Thanks to this analysis, we have identified and summarize three key issues that lead us to build a protocol:

- Map background

- Nonlinear text placement

- Toponymic hierarchies

The first two points are the main phenomena that disturb reading conditions. They are simulated and treated individually at first. First, we use this method with DTP software like Illustrator. We then transposed these tests into m.s.s. using TileMill, which allowed us to test this protocol with a more parametric/programmatic logic. This step allowed us to understand how text could be styled and parametrized in a GIS environment. Therefore it facilitated our learning of the construction of a more complex cartographic environment and the manipulation of geographic data. The third point brings another dimension to the previous categories in connecting issues relatives to the anatomic structure of the font face to the distribution problematic:

\subsubsection{Map background}

A toponym is rarely displayed on a white and uniform background. On the opposite, it rather can be dark and heterogeneous background. Moreover text can overlay elements from the same color or structure as buildings or roads. There is a need to anticipate the overlay phenomena that may arise. Place names can be superimposed to an area with a high-density contrast (for instance urban areas), moving from darkness to lights (for instance from one mountain slope to the other), or pass through an area of great formal complexity (for instance rocky mountains textures). What typographical structures can solve this? How to isolate the letters? What kind of methodological mechanisms can be used in type design to help letterforms to stand out from the map background?

We designed various canvases as a whole system or program to simulate graphic and visual phenomena that may arise (Figure 2). They reproduce in some way the conditions that can be encountered by the place names on the cards. This graphic simulation includes dot, line area elements that usually disturb the reading of toponyms.

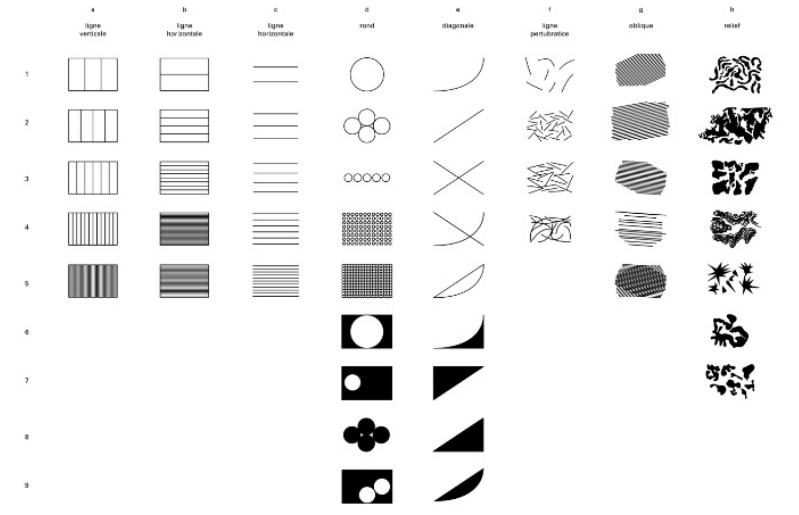

Fig. 2. Typographic standards/specifications of IGN. 1: Type 1972, 2: Type 1993, 3: Type 1922.

\subsubsection{Nonlinear text placement}

Which structure is best adapted to the fact that letters are typeset in curved situations? How letters and words do they behave when the baseline follows the most winding and steep lines? Regarding toponyms, there are two broad categories: those composed horizontally (the localities) and those that can be described as "layout place names" which are typeset according to surfaces or lines (ex: forests, mountain ranges, geographical areas, roads and water-courses). How do letters and words behave, when their baseline is as winding and steep as the landforms?

The curve repository (Figure 3) has been designed in a similar process that the one used to deal with the issue of map background. This repository makes it possible to know what kind of curve and rhythm are the most difficult to manage for non linear text placement.
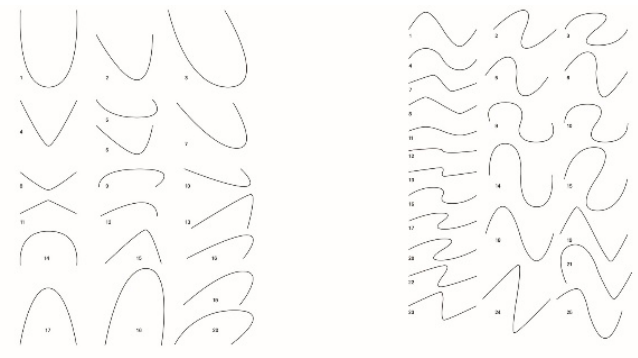

Fig. 3. A curve repository.

In a more prospective approach, we tried to simulate how the structure of letterforms could behave and change when the baseline (on which characters are set) is twisted. (Figure 4) We have therefore simulated various situations by deforming the glyphs space according to the angle that the baseline adopts.

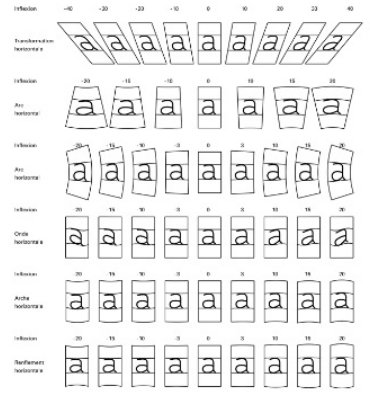

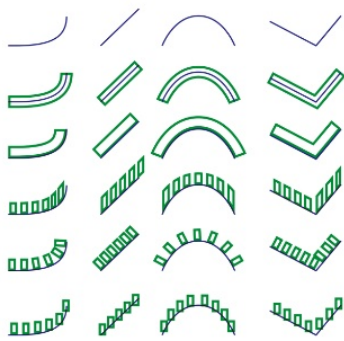


Fig. 4. A prospective approach: deformation of the glyph space.

Although this approach was subsequently ruled out because it cannot yet be technically exploited, it may be of interest to consider this approach to the problem.

\subsubsection{Toponymic hierarchies}

One of the lessons learnt from the analysis of our documented corpus and especially specifications, is the framework of text hierarchies necessary to issue the topographic message.

How (and how often) different reading levels coexist and allow to build and make the chart readable message? To create useful fonts, type designers need to consider not only how characters relate to each other within a style, but also how different styles relate to each other within a font family. In the case of a character designed for topographic maps, each style has to be related to a topographic function.

\subsection{The type design process}

It was first envisaged to draw a skeleton (Figure 5) for providing a structure that could anticipate deformations.
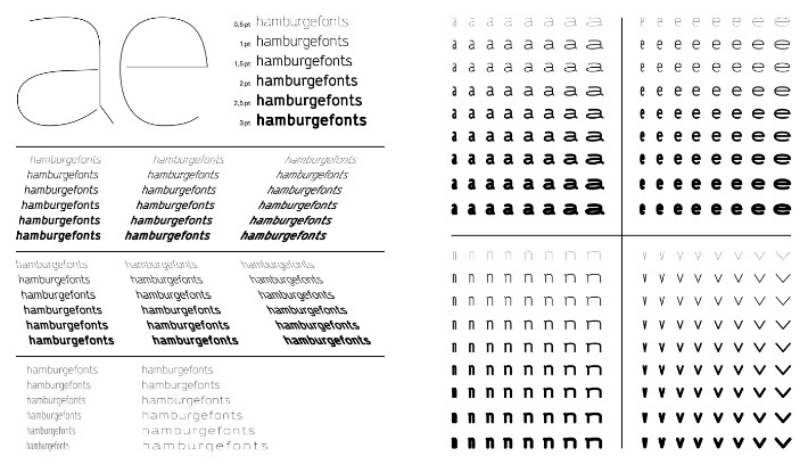

Fig. 5. The skeleton process.

This step was the starting point for the design of several versions. The resulting family (Figure 6), designed in four styles, is based on the textual standards that have been implemented by the French national mapping agency (IGN). Two typefaces were designed for populated places. The first one is rather narrow in order not to interfere with the background's legibility; the second one is more legible at small sizes. The others were designed for natural places. The italic version suits for the oronyms \& the hydronyms (mountains \& watercourses). The serif version is used for uninhabited place names.

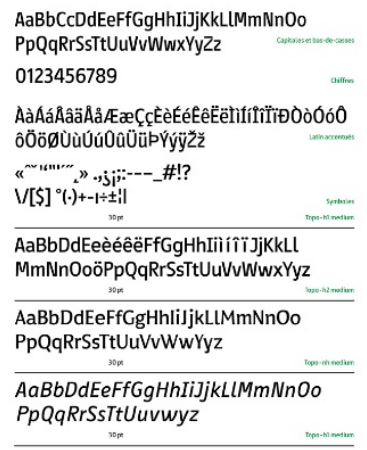

Fig. 6. Distribution of the family according to the specifications.

\section{Perspectives and further work}

This protocol and the typographic design have been developed jointly and in some respects typefaces have not been fully developed using the protocol. The typographic research undertaken as part of this project has brought some guidelines and elements which provide a substantive basis for further research. It provides insight on theses specific questions, and raises new ones.

Even if this approach has been developed in a first time independently outside of the academic research context and the cartographic research, we truly believe that this method and type design in general has a key role to increase legibility in map design and can be fruitful for readability especially when it comes to addressing dynamic display issues. But further research is necessary to improve this kind of approach. This is why we decide to take this re-search further.

The project is continued by a starting $\mathrm{PhD}$ co-supervised by IGN, the French national mapping agency, ESAD Valence and ANRT. The aim is to seek possible harmony between programmatic type design and dynamic multiscale mapping applications.

Typefaces are traditionally seen as static letter shapes. Very recently the development of variable fonts, as part of OpenType 1.8 (Hudson 2016) and parametric type design tools enable the lettershapes to leave their static paradigms adapted of the digital stream (Babé 2016). Their setting in motion, their animation, disrupts their definition. These recent technical implementations in the field of type design industry allow us to adapt fonts to a precise con-text. Moreover it link these different setting by making letterforms move. We believe that environmental constraints of dynamic mapping systems are certainly the richest places if we want to manage huge adaptive textual systems. Therefore we will use the foundations of the project presented here and push them further to explore the exciting challenges of the latest technical innovations in type design.

\section{Acknowledgements}

Our work is funded in part by the ESAD Grenoble/Valence. The authors thank the ICA for providing the grant that helped us register and attend this conference.

\section{References}

Babé, L.R. (2016) https://www.prototypo.io/blog/versatile-type-design-forthe-web.html. Accessed 28 Oct 2016.

Barrault, M. (2001). A methodology for placement and evaluation of area map labels. Computers, Environment and Urban Systems, 25(1), 33-52.

Bartz, S. (1970). An Analysis of the Typographic Legibility Literature: Assessment of its Applicability to Cartography. The Carto-graphic Journal of Film Writing, 7(1), 10-16. 
Hudson, J. (2016). Introducing OpenType Variable Fonts. https://medium.com/@tiro/https-medium-com-tirointroducing-opentype-variable-fonts-

12ba6cd2369\#.mzfpgln42. Accessed 21 Sep 2016.

Noordzij, G. (2000). Letterletter. Hartley and Marks Publishers (pp. 30). Vancouver.

Noordzij, G. (2005). The Stroke. Hyphen Press (pp. 9). London. 